\title{
Are Th17 cells in the gut pathogenic or protective?
}

\author{
A Symons ${ }^{1}$, AL Budelsky $^{1}$ and JE Towne ${ }^{1}$
}

\section{Th17 cells are abundant in multiple chronic inflammatory and autoimmune diseases. Clinical trials with antibodies to interleukin (IL)-17A, one of the Th17-cell signature cytokines, have recently reported therapeutic benefit in multiple patient populations; however, in Crohn's disease the role of Th17 cells and IL-17A appears to be more complicated. The development of different subsets of Th17 cells and their relative pathogenic activities with a focus on the gut environment will be discussed.}

Th17 cells are $\mathrm{CD} 4^{+} \mathrm{T}$ lymphocytes, which not only have an important role in host defense to extracellular bacteria and fungi but have also been described as uniquely pathogenic in multiple inflammatory diseases. Th17 cells produce a number of signature cytokines including, but not limited to, interleukin (IL)-17A, IL-17F, IL-22, and IL-21, and require expression of the transcription factor ROR $\gamma \mathrm{t}$ for development and IL23 for maturation and expansion. Mouse models of inflammation provided the first evidence that IL-23-driven Th17 cells are pathogenic; in that, deficiency of IL-23 was protective and associated with reduced IL-17A at sites of immune pathology. Furthermore, mice lacking IL-17A or IL-17RA, one of its receptor subunits, showed reduced disease in the experimental autoimmune encephalomyelitis (EAE) model of multiple sclerosis, the collagen-induced arthritis model and in mouse models of psoriasis ( $\mathrm{PsO}$ ). These preclinical data provided support for a critical role for Th17 cells and IL$17 \mathrm{~A}$ in the pathology associated with multiple inflammatory diseases. Other immune cell types in particular innate lymphoid cells and $\gamma \delta$ T cells have subsequently been identified as additional major sources of IL-17A, although their role in disease is currently less clear. ${ }^{1}$

Recently, data for the role of IL-17A in human disease has emerged. Secukinumab (AIN457) is a fully human neutralizing antibody to IL-17A and beneficial clinically relevant responses have been reported in patients with $\mathrm{PsO}$, rheumatoid arthritis (RA), and uveitis after treatment. ${ }^{2}$ Similar improved symptoms were seen in RA patients treated with another IL-17A therapeutic, LY2439821, a humanized monoclonal antibody to IL-17A. ${ }^{3}$ Taken together these clinical data provide the first evidence that IL-17A has a significant role in the pathophysiology of human disease.

The definition of Th17 cells as a single lineage has been brought into question with the description of heterogeneous populations of IL-17A-producing CD4 ${ }^{+}$ $\mathrm{T}$ cells with various degrees of pathogenicity. ${ }^{4,5}$ Murine Th17 cells differentiated in the presence of transforming growth factor- $\beta$ express more CCL2 0 and IL-10, whereas Th17 cells differentiated in the absence of transforming growth factor$\beta$ and in the presence of IL- 1 , and IL-23 express Tbx21 (T-bet), the master regulator of Th1 cells and interferon (IFN) $-\gamma$ along with IL-17A. In adoptive transfer experiments, IL-23-driven Th17 cells were more pathogenic than Th17 cells differentiated in the presence of transforming growth factor- $\beta{ }^{4}$ Other Th 17 cells generated in vitro have been described that also express cytokines classically associated with Th2 cells or a regulatory T-cell phenotype. ${ }^{6}$ In addition, not only can Th 17 cells express other cytokines at the same time as IL-17A they can also stop expressing IL-17A altogether. Recent work with fate-mapping knock-in mice showed that IL-17A expression by $\mathrm{CD} 4^{+}$cells can be transient and these so-called ex-Th17 cells can acquire the ability to express IFN- $\gamma$ and lose the ability to express IL-17A under certain conditions. ${ }^{7}$ In the inflamed spinal cords of mice with EAE, T cells expressing IFN $-\gamma$ predominantly originated from cells that previously produced IL-17A and this conversion was IL-23dependent, whereas in an acute cutaneous infection model, T cells making IL-17A neither lose expression of IL-17A nor did these cells acquire expression of other cytokines. ${ }^{7}$ DNAse sensitivity studies and global mapping of epigenetic markers of active and closed chromatin help explain the potential for plasticity at the molecular level as the IFN- $\gamma$ and Tbx21 loci are poised for expression in Th17 cells differentiated in vitro. ${ }^{8}$ It appears then that the local environment and cytokine milieu at sites of inflammation are key factors in both the development of different types of Th17 cells and their conversion into distinct subtypes with various levels of pathogenic activity. 
The identification of distinct Th17 cell populations may be particularly relevant in the gut. Despite the clear pathogenic activity of Th17 cells in mouse models of $\mathrm{PsO}$, arthritis, and neurodegeneration, Th17 cells and IL-17A have been shown to have both pathogenic and tissue-protective roles in animal models of colitis. In chemically induced colitis models, some Th17 cell cytokines such as IL17A and IL-22 appear to be protective, whereas others such as IL-17F and IL-21 are pathogenic. ${ }^{5}$ In the T-cell transferinduced mouse colitis model in which donor $\mathrm{CD} 45 \mathrm{RB}^{\text {high }} \mathrm{CD} 4^{+} \mathrm{T}$ cells are transferred into $\mathrm{Rag}^{-/-}$recipients, transfer of IL-17A ${ }^{-/-} \mathrm{T}$ cells results in severe colitis, suggesting that IL-17A is dispensable for colitis induction. ${ }^{5}$ Although several studies have demonstrated that colitis induced by IL-17 $\mathrm{A}^{-1-} \mathrm{T}$ cells is indistinguishable from that induced by wild-type $\mathrm{T}$ cells, one study examined mice earlier in the disease process and demonstrated worse pathology in recipients of IL$17 \mathrm{~A}^{-/-} \mathrm{T}$ cells that was associated with increased Th1 gene expression including IFN $-\gamma .{ }^{9}$ These data support a protective role for T-cell-derived IL-17A in this T-cell transfer-induced colitis model. Transfer of IL-23R $\mathrm{R}^{-/} \mathrm{T}$ cells did not trigger colitis and this was attributed to the failure of IFN- $\gamma / \mathrm{IL}-17 \mathrm{~A}$ double producing cells to develop in the absence of IL-23 signaling, consistent with IL-23driven Th17 cells being pathogenic.

A recent report provides new insight into why different subsets of Th17 cells in the gut may have contradictory roles. Esplugues et al. ${ }^{10}$ describe how proinflammatory Th17 cells can be reprogrammed in the gut to take on a regulatory function. Primarily using an anti-CD3 antibody-induced mouse model of tolerance, they first demonstrate that Th17 cells accumulate in the small intestine in response to a strong T-cell receptor stimulus. Th17 cell accumulation is dependent on the CCL20/CCR6 chemokine/chemokine receptor axis as well as on IL-17A/F signaling in a positive feedback loop. The severity of intestinal pathology in response to antiCD3 antibody treatment correlates with accumulation of Th17 cells in the gut but the disease is transient. Remarkably, the authors found that although some cells are eliminated via the intestinal lumen, the remaining Th17 cells in the duodenum acquire immunosuppressive properties. When the expression profile of these regulatory Th17 (rTh17) cells, which

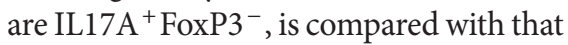
of Th17 cells from the central nervous system from mice with EAE, both Th17 cell populations have similar activation markers (CD25, CD69), but rTh17 cells have less proinflammatory tumor necrosis factor- $\alpha$ and IL-2 and more anti-inflammatory IL-10. In fact, the suppressive capacity of the rTh17 cells is dependent on IL-10, transforming growth factor- $\beta$, and CTLA-4. Th17 cells do not accumulate in the gut in CCR6-deficient mice and are increased in the spleen and lymph nodes relative to wild-type mice. Furthermore, splenic Th17 cells from CCR6-deficient mice do not have suppressive activity implying migration to the small intestine is necessary for the acquisition of regulatory function. The mechanism underlying the conversion of proinflammatory Th17 cells to rTh17 cells remains to be elucidated but correlates with migration of the cells into the gut, suggesting a unique microenvironment exists in this tissue to facilitate Th17 cell control.

Increased numbers of Th17 cells are found in the inflamed mucosa of patients with Crohn's disease (CD) and ulcerative colitis as compared with normal colonic mucosa, and it has become clear that similar to the mouse models, Th17 cells found in the gut of Crohn's disease patients are also heterogenic. Several studies have identified a subset of Th17 cells isolated from gut mucosa of CD patients that produce IFN- $\gamma$ along with IL-17A. ${ }^{11,12}$ In addition, a subset of Th17 cells isolated from the lamina propria of $\mathrm{CD}$ patients has been reported to express FoxP3 and exhibit suppressive activity. ${ }^{6}$ Therefore, there is overlap among Th17, Th1, and Treg cell lineages in the colonic mucosa of CD patients, and this heterogeneous population of gut Th17 cells that express IL-17A in combination with either IFN- $\gamma$ or FoxP3 likely contains cells with different, potentially opposing roles. Recently, results from a proof-ofconcept study with secukinumab demonstrated that blockade of IL-17A was ineffective in patients with Crohn's disease and in post-hoc analyses it appeared that secukinumab in fact exacerbated disease in a subset of patients. ${ }^{13}$ These results contrast with the efficacy reported with IL-17A inhibitors in small populations of RA, PsO, and non-infectious uveitis patients, providing additional support for a complex and potentially unique role for IL-17A and Th17 cells in the gut.

In summary, Th17 cells, defined by expression of IL-17A, are abundantly present in multiple chronic inflammatory and autoimmune diseases such as $\mathrm{PsO}, \mathrm{RA}$, and inflammatory bowel disease. However, recent clinical data examining the efficacy of IL-17 inhibitors in multiple patient populations has called into question whether Th17 cells are similarly pathogenic across different diseases. Clinical trials with antibodies to IL-17A have reported benefit in $\mathrm{PsO}$, $\mathrm{RA}$, and uveitis patient populations, whereas in Crohn's disease no clinical benefit and some exacerbation of disease was observed, suggesting the role of Th17 cells and IL-17A in inflammatory bowel disease is different from other inflammatory diseases. It is clear that different subsets of Th17 cells exist in the gut mucosa. $\mathrm{CD} 4{ }^{+} \mathrm{T}$ cells expressing both IFN- $\gamma$ and IL-17A appear to represent the pathogenic sub-population of Th17 cells, whereas rTh17 cells with suppressive properties may be protective, although further characterization of the relationships between these populations is required. The new study from Esplugues et al. ${ }^{10}$ suggests something specific about the gut environment drives Th17 cells to acquire a protective function. Perhaps the unique exposure to innate signals in the gut due to the intense bacterial load creates a suppressive environment. Specific taxon of microbiota have been shown to influence the generation of Th17 cells in the intestine and it would be interesting to evaluate whether the makeup of the commensal microbiome is involved in the transition of proinflammatory Th17 cells to rTh17 cells. ${ }^{14}$ It is also possible that IL-17A has gut-specific homeostatic roles such as promoting the integrity of the epithelial cell barrier and regulating growth of certain pathogens. Clearly, the role for Th17 cells and IL-17A in the gut 
is more complex than originally hypothesized, and continued research, investigating the different Th17 cell populations and their respective functional roles, as well as the role of innate sources of IL-17A in this tissue, is required.

\section{DISCLOSURE}

All authors are employees and stockholders of Amgen.

(C) 2012 Society for Mucosal Immunology

\section{REFERENCES}

1. Spits, H. \& Di Santo, J.P. The expanding family of innate lymphoid cells: regulators and effectors of immunity and tissue remodeling. Nat Immunol 12, 21-27 (2011).

2. Hueber, W. et al. Effects of AIN457, a fully human antibody to interleukin-17A, on psoriasis, rheumatoid arthritis, and uveitis. Sci. Transl. Med. 2, 52 ra72 (2010).
3. Genovese, M.C. et al. LY2439821, a humanized anti-interleukin-17 monoclonal antibody, in the treatment of patients with rheumatoid arthritis: a phase I randomized, double-blind, placebocontrolled, proof-of-concept study. Arthritis and Rheumatism 62, 929-939 (2010).

4. Ghoreschi, K., Laurence, A., Yang, X.P., Hirahara, K. \& O'Shea, J.J. T helper 17 cell heterogeneity and pathogenicity in autoimmune disease. Trends Immuno/ 32, 395-401 (2011).

5. Morrison, P.J., Ballantyne, S.J. \& Kullberg, M.C. Interleukin-23 and Thelper 17-type responses in intestinal inflammation: from cytokines to T-cell plasticity. Immunology 133 , 397-408 (2011).

6. Hovhannisyan, Z., Treatman, J., Littman, D.R. \& Mayer, L. Characterization of interleukin17-producing regulatory $T$ cells in inflamed intestinal mucosa from patients with inflammatory bowel diseases. Gastroenterology 140, 957-965 (2011)

7. Hirota, K. et al. Fate mapping of IL-17producing $\mathrm{T}$ cells in inflammatory responses. Nat Immunol 12, 255-263 (2011).
8. Wei, G. et al. Global mapping of H3K4me3 and $\mathrm{H} 3 \mathrm{~K} 27 \mathrm{me} 3$ reveals specificity and plasticity in lineage fate determination of differentiating CD4+ T cells. Immunity $\mathbf{3 0}$, 155-167 (2009).

9. O'Connor, W. Jr. et al. A protective function for interleukin 17A in T cell-mediated intestinal inflammation. Nat Immunol 10, 603-609 (2009).

10. Esplugues, E. et al. Control of TH17 cells occurs in the small intestine. Nature 475, 514-518 (2011).

11. Annunziato, F. et al. Phenotypic and functional features of human Th17 cells. J Exp Med 204, 1849-1861 (2007).

12. Sarra, M., Pallone, F., Macdonald, T.T. \& Monteleone, G. IL-23/IL-17 axis in IBD. Inflamm Bowel Dis 16, 1808-1813 (2010).

13. Hueber, W. et al. Inhibition of IL-17A by secukinumab is ineffective for Crohn's disease (CD). J Crohns Colitis 5, S7 (2011).

14. Ohnmacht, C. et al. Intestinal microbiota, evolution of the immune system and the bad reputation of pro-inflammatory immunity. Cell Microbiol. 13, 653-659 (2011). 